Pensamiento Crítico No. 7, pág. 157 - 172

\title{
En El Perú se Pueden Modelar y Predecir los Rendimientos Accionarios?
}

Jorge Barrera $\mathrm{H}$.

Resumen: Las redes neuronales artificiales (RNA) se han convertido en un importante instrumento para modelar y predecir los rendimientos accionarios y por ende para evaluar la eficiencia del mercado de capitales (HEM). Debido a que son modelos que incorporan variables no lineales (característica de la mayoría de las series económicas y financieras) funcionan mejor que los modelos estadísticos tradicionales, como las regresiones lineales o modelos Box-Jenkins (Camino Aleatorio).

Es este artículo se dan algunos alcances sobre investigaciones de verificación de las hipótesis de eficiencia de los mercados de capitales y las ventajas de la utilización de los modelos de redes neuronales artificiales (RNA), principalmente para la predicción de tendencias de precios.

Palabras claves: Mercados de capitales, Hipótesis de eficiencia de los mercados de capitales, Caminata aleatoria, Finanzas conductivistas, Algoritmos genéticos, Redes neuronales artificiales. 


\section{Jorge Barrera H.}

\section{CUAL ES LA SITUACIÓN}

Hace más de dos siglos que Adam Smith proclamó que quienes buscaban su propio provecho en una economía competitiva promovían más eficazmente el interés público.

Esto se ha constituido en una de las ideas más discutidas y consensuadas de la historia, al determinar que el mercado de competencia perfecta es el más eficiente asignador de recursos, fuerza que eleva el nivel de vida y el de la producción.

La eficiencia tal como la conciben los economistas, es un proceso por el cual la sociedad extrae de los recursos existentes la máxima cantidad de satisfacción para los consumidores. Esto se conoce como la eficiencia en el sentido de Pareto, en la que no es posible mejorar el bienestar de ninguna persona sin empeorar el de alguna otra.

Cada vez más, el consenso mundial le da hoy en día a los mercados en general y a los mercados de capitales en particular, una mayor importancia. La cual es resaltada por la globalización, que ha liberalizado los mercados financieros principales, por los avances en las tecnologías de las telecomunicaciones y computación y por la institucionalización de dichos mercados.

Desde el hoy famoso enunciado de Keynes (1936) acerca de que la mayoría de las decisiones de los inversionistas "puede sólo ser tomadas como resultados de espíritus animales, es decir, de un impulso espontáneo de actuar más que de permanecer quietos, y no como resultado de un promedio ponderado de beneficios multiplicados por probabilidades cuantificadas", muchas investigaciones han sido dedicadas a examinar la eficiencia en la estructura de precios de mercado de las acciones

En 1970, Fama definió un mercado eficiente (hipótesis de mercado eficientes) como aquel en el cual, los precios siempre reflejan "plenamente" toda la información disponible, en su investigación principal establece que los rendimientos de los activos financieros, con particular hincapié en las acciones, no pueden ser predecibles. Es aquí que nace la noción de la hipótesis de mercados eficientes (HEM).

Por ello, las siguientes serían condiciones suficientes para un mercado eficiente:

1.- Inexistencia de costos de transacción.

2.- Toda la información disponible se puede obtener sin costo por todos los participantes en el mercado.

3.- Todos los participantes en el mercado tienen igual horizonte de tiempo y expectativas homogéneas respecto a los precios. 
En el Perú se pueden modelar y predecir los rendimientos accionarios?

En un mercado eficiente, los precios deben reflejar toda la información disponible y deben ajustarse rápidamente a la nueva información. Así, un mercado eficiente supone la existencia de condiciones de un mercado perfectamente competitivo, el comportamiento racional de quienes operan en dicho mercado y las condiciones de gratuidad informacional.

Es difícil que en los mercados se den todas estas condiciones. Como dice Fama (1970), estas condiciones son suficientes pero no necesarias, pues si el número de participantes con acceso a la información disponible es lo suficientemente amplio, ninguno de ellos puede consistentemente analizar de forma más atinada que los otros la información existente, y los costos de transacción, aunque altos, son conocidos y tenidos en cuenta por los participantes; así, las condiciones anteriores no se cumplen en el sentido estricto, pero el mercado ciertamente será eficiente.

Roberts (1967) definió tres niveles de eficiencia, (HEM) en función del conjunto de información que se considera incorporada en el precio. Estos niveles de eficiencia los denominó: débil, semifuerte o intermedio y fuerte.

Hipótesis de Eficiencia débil: el precio incluye toda la información histórica.

La conclusión es que no se puede ganarle al mercado sistemáticamente, utilizando sólo información histórica.

Con esta definición está anulándose la posibilidad de que utilizando análisis técnico se pueda obtener de forma sistemática rentabilidad extraordinaria.

Hipótesis de eficiencia semifuerte o intermedia: el precio incluye toda la información histórica y pública.

En conclusión no se le puede ganar al mercado, de forma sistemática, utilizando información histórica y pública.

Con esta definición está anulándose la posibilidad de que, utilizando análisis fundamental se pueda obtener de forma sistemática rentabilidad extraordinaria.

Hipótesis fuerte: el precio incluye toda la información histórica y toda la información pública y privada.

Sistemáticamente no es posible ganarle al mercado, de acuerdo con la hipótesis de eficiencia fuerte, utilizando toda la información disponible, sea esta pública o privada. 


\section{Jorge Barrera H.}

Con esta definición se está anulando la posibilidad de que, utilizando cualquier tipo de análisis o técnica se pueda obtener rentabilidad extraordinaria.

Para que se cumpla la eficiencia de mercado en cada una de sus hipótesis, los operadores del mismo han de utilizar necesariamente todo el conjunto de información disponible, según la hipótesis considerada, porque solo de este modo se está incorporando la información en el precio. Esto significa que al realizar masivamente análisis técnico, fundamental o de cualquier otra clase, se contribuye a que el mercado gane en eficiencia.

Es el manejo de la información lo que determina la eficiencia del mercado y los grados de esta eficiencia dependerán de que no se puedan obtener beneficios poco comunes o excesivos mediante el uso de dicha información.

Sin conocer nada especial acerca de un activo financiero, un inversionista en un mercado de capitales eficiente, espera obtener una rentabilidad de equilibrio de su inversión y una compañía espera pagar un costo de capital también de equilibrio, se debería demostrar además que si el mercado financiero es eficiente los gerentes corporativos no podrían generar valor engañando a los inversionistas.

La economía peruana, durante las últimas décadas, ha seguido un esquema de desarrollo basado en un modelo de mercado, orientado a la apertura del comercio internacional, con un sector minero exportador fuerte y en el que al mercado se le ha entregado el papel preponderante en la asignación de los recursos. En el mercado de capitales peruano la participación de los Inversionistas Institucionales, así como las Administradoras de Fondos de Pensiones, AFPs, son los que han logrado ocasionar un mayor desarrollo, dinámica yeficiencia de este.

\section{CUALES SON LAS PREGUNTAS QUE DEBEN SER RESPONDIDAS}

Las preguntas que hay que hacerse para evaluar el nivel de eficiencia de nuestro mercado de capitales son:

¿En nuestro mercado de capitales peruano los precios corrientes reflejan la información disponible?

Si la respuesta es afirmativa, entonces nuestro mercado de capitales es un mercado eficiente.

¿En el Perú se pueden modelar y predecir los rendimientos accionarios? 
En el Perú se pueden modelar y predecir los rendimientos accionarios?

¿En el Perú pueden crear valor las decisiones de financiación?

Si la respuesta es negativa, entonces nuestro mercado de capitales es un mercado eficiente.

\section{CONSECUENCIAS DE LA EXISTENCIA DE UN MERCADO EFICIENTE}

Si nuestro mercado peruano es eficiente, entonces esto significaría que los precios de mercado corriente reflejan el valor actual implícito de los títulos y no es posible tener beneficios poco comunes o excesivos usando la información disponible, no pudiendo existir oportunidades de financiación con valor presente neto positivo

Esto eliminaría la pertinencia de muchas estrategias de incremento de valor de las empresas en la toma de decisiones de financiación, tales como: no podrían programar las emisiones de acciones y obligaciones, podrían vender tantas acciones y obligaciones como deseen sin temor a una reducción del precio, y los mercados de acciones y obligaciones, no se verían afectados por las empresas que camuflen información contable interesadamente.

Resumiendo, esto significaría que los gerentes no podrían crear valor engañando a los inversionistas, dado que todos los títulos siempre serán tasados de manera pertinente por lo inversionistas.

\section{ALGUNAS IDEAS ERRONEAS ACERCA DE LA HIPÓTESIS DEL MERCADO EFICIENTE. (HEM)}

Es como lanzar dardos: Cuando se divulgó la idea de mercado eficiente, esta se caracterizó por la cita siguiente: “ ....lanzar dardos a la página financiera, producirá una cartera de la que se puede esperar que tenga un comportamiento tan bueno como el de cualquier cartera organizada por los analistas profesionales de títulos." 1 Esto no es completamente cierto. Lo que hay que entender es que, en promedio, el gerente no podrá lograr una rentabilidad anormal o excesiva, con respecto a alguna rentabilidad esperada de referencia que provenga de la línea del mercado de valores, generada por el modelo de asignación de precios de activos de capital, MAPAC.

Lo que significa la eficiencia de mercado es que el precio que una empresa obtendrá cuando venda una acción es un precio justo, porque este refleja el valor del capital considerando la información disponible acerca del mismo. 
Jorge Barrera H.

Inconsistencia entre las fluctuaciones de precios y la eficiencia: Justamente una acción se ajusta a la nueva información cambiando de precio. Por el contrario, en un mundo globalizado, la falta de movimiento de los precios podría ser indicio de ineficiencia.

Pocas acciones circulantes cambian de manos de un día para otro: ¿̇s señal de eficiencia el hecho de que muy pocas acciones cambien de manos en un día determinado? Esto más bien parece ser una señal de ineficiencia, sin embargo, el número de negociadores de una acción en un día determinado, por lo general es bastante menor que el de las personas que la tienen. Esto sucede así porque un individuo sólo negociará cuando su estimación del valor del título difiera lo suficiente del precio de mercado para justificar incurrir en comisiones de corretaje y otros gastos de transacción. También, si el número de personas que comercia una acción es reducida en comparación con el número de acciones en circulación, se puede esperar que la acción sea valorada de manera eficiente en tanto que los negociantes interesados usen la información disponible al público. Es decir, el precio de la acción puede reflejar la información disponible al público, aun si muchos accionistas no tienen la acción y no están considerando negociar en un futuro próximo.

\title{
MODOS PRINCIPALES DE EVALUAR LAS HIPOTESIS DE EFICIENCIA. (HEM)
}

\section{Series de tiempo. Modelos lineales de estimación de nivel.}

\begin{abstract}
a) Proceso estocástico estacionario. Modelos autorregresivos según la metodología Box - Jenkins: buscan patrones de estacionalidad y de predicibilidad en el tiempo.
\end{abstract}

De la misma manera como se utiliza información muestral para inferir sobre una población, en las series de tiempo se utiliza la realización para inferir sobre el proceso estocástico subyacente.

Un tipo de proceso estocástico que ha recibido gran atención y que han sido objeto de escrutinio por parte de los analistas de series de tiempo, es el llamado proceso estocástico estacionario.

En términos generales, se dice que un proceso estocástico es estacionario si su media y su varianza son constantes en el tiempo y si el valor de la covarianza entre dos períodos depende solamente de la distancia o rezago entre estos dos períodos de tiempo y no del tiempo en el cual se ha calculado la covarianza. 
En el Perú se pueden modelar y predecir los rendimientos accionarios?

Un modelo, que es un proceso estacionario que converge sobre la media, es el Modelo Random Walk (camino aleatorio)

\section{ARIMA o modelo Random walk (Camino Aleatorio)}

Este es uno de los modelos utilizados para contrastar la hipótesis de la eficiencia del mercado (HEM), en su forma débil.

Según este modelo, los precios bursátiles siguen un proceso aleatorio con un elemento constante, o drift.

Donde:

$$
\mathrm{P}_{\mathrm{t}}=\mathrm{P}_{\mathrm{t}-1}+\mu+\varepsilon_{\mathrm{t}}
$$

a) $\mathrm{P}_{t}$ y $\mathrm{P}_{\mathrm{t}-1}$ son los precios acaecidos (ex post) de hoy y ayer

b) es la rentabilidad esperada de la acción, y es una constante $\mu$

c) $\varepsilon_{\mathrm{t}}$ es un elemento aleatorio con las siguientes características:

- La esperanza matemática de $\varepsilon_{\mathrm{t}}$ es cero. En media, los $\varepsilon_{\mathrm{t}}$ de cada período serán cero.

- La covarianza de varios $\varepsilon_{\mathrm{t}}$ de diversos períodos es cero. Es decir, los $\varepsilon_{\mathrm{t}}$ de diversos periodos son independientes entre sí.

- La varianza del elemento aleatorio es $\sigma^{2}$, que a su vez es también la varianza de los precios, yaque $\mathrm{P}_{\mathrm{t}-1}$ y son constantes. $\mu$

- El elemento aleatorio de hoy es independiente del precio de ayer.

¿Cómo se generan las rentabilidades a partir del modelo Random Walk.? La rentabilidad es la diferencia de precios de una acción entre dos períodos $\mathrm{t}$ y $\mathrm{t}-1$.

$$
\mathrm{E}\left(\mathrm{R}_{\mathrm{t}+1}\right)=\mathrm{E}(\mu)+\mathrm{E}\left(\varepsilon_{\mathrm{t}+1}\right)=\mu+0=\mu
$$

Si queremos estimar el valor de la rentabilidad mañana $E\left(R_{t+1}\right)$ teniendo en cuenta la rentabilidad obtenida hoy $\left(R_{t}\right)$, obtenemos la esperanza matemática de la expresión: 
Jorge Barrera H.

$$
\mathrm{E}\left(\mathrm{R}_{\mathrm{t}+1}\right)=\mathrm{E}(\mu)+\mathrm{E}\left(\varepsilon_{\mathrm{t}+1}\right)=\mu+0=\mu
$$

Es decir, nuestra mejor estimación de la rentabilidad de mañana, o de pasado mañana, o de cualquier día futuro, es siempre una constante $\mu$, que es la rentabilidad esperada de la acción para cada período. Es por ello que este proceso de generación de rentabilidades se denomina proceso estacionario que converge sobre la media.(si el modelo es cierto).

Pero cualquiera que sea la forma como escribamos el modelo, este no es estacionario. ${ }^{2}$ Si se diferencia una vez se obtiene el modelo transformado, el cual siempre es estacionario (proceso no estacionario homogéneo de primer grado). Por sustituciones sucesivas revertimos la situación, o lo que es lo mismo, integramos el proceso denominado proceso autorregresivo-medias móviles integrado o ARIMA $(0,1,0)$

Las pruebas típicas de estacionariedad deben descartar en primer lugar que $\mathrm{P}_{\mathrm{t}}$ (ARIMA) sea estacionaria en niveles (lo que supondría una forma de predicibilidad o eficiencia débil), debiendo ser estacionaria en diferencias. En segundo lugar, se deberá descartar que el término de error contenga algún componente de autocorrelación temporal (también supondría algún grado de predicibilidad)

\section{b) Test de estrategias}

Consiste en plantear una estrategia de carteras (tales como inversión en pequeñas empresas, en empresas con PER bajo o con alta rentabilidad por dividendos, etc.) y comprobar sus resultados. Si estos son superiores a los que ha obtenido el mercado (estrategia pasiva de comprar y mantener), se puede decir que el mercado no es eficiente, pues hay determinada información pública que no está incluida en los precios y que es usada solo por los que utilizan la estrategia; esta información "extra" les permite alcanzar rentabilidades anormalmente grandes.

\section{c) Estudios de eventos}

También se analiza el efecto producido en la cotización por determinadas informaciones sobre la situación de la empresa, tales como anuncios de dividendos, fusiones, etc. Es lo que se conoce como estudios de eventos en el mercado. Sirve para evaluar la eficiencia a nivel semi fuerte (de un split accionario)

\section{d) Estudios de cointegración entre mercados}

Si dos mercados están cointegrados entonces estos mercados no son eficientes. 


\section{En el Perú se pueden modelar y predecir los rendimientos accionarios?}

\section{EL TEMA DE LA INEFICIENCIA EN LOS MERCADOS FINANCIEROS}

Varios estudios han concluido que existe suficiente evidencia de que los precios accionarios en mercados financieros desarrollados y emergentes no siguen un camino aleatorio $^{3}$ y muestran que los retornos accionarios son predecibles en algún grado, pues la presencia de autocorrelación en las distintas series analizadas y, ciertamente, los retornos no siguen una distribución definida, independiente e idéntica; mucho menos una distribución normal. La diferencia entre mercados desarrollados y emergentes radica mas bien en la magnitud de la dependencia serial, que por ser pequeña no permite la obtención de ganancias extraordinarias en los primeros.

Samuelson (1998) afirma, el mercado bursátil es micro-eficiente pero macroineficiente, es decir, que la hipótesis de la eficiencia del mercado se cumple mejor para acciones individuales que para los índices de precios agregados del mercado.

Es posible, que en el tiempo y por la globalización, el diferencial entre mercado emergente (menos eficiente) y mercados desarrollados se vaya desvaneciendo hasta un punto en el que se pueda concluir que, aunque es cierto que se presenta un cierto grado de dependencia serial, su magnitud es tan pequeña que no permite un aprovechamiento económico y, por lo tanto, el mercado se acerca al ideal de mercado eficiente.

Un aspecto controversial de estos resultados es si esta predicibilidad expost es una señal de ineficiencia del mercado. La predicibilidad puede bien ser consecuencia de cambios sistemáticos en la tasa de descuento o cambios en el factor prima por riesgo, que no pueden ser captados por los modelos utilizados. ${ }^{4}$ No es que los precios sean estrictamente predecibles, sino que dichos precios dependen de ciertos factores de riesgo exógeno, cuya evolución puede generar determinados patrones de comportamiento en los precios. En tal sentido, puede que los mercados sean eficientes y los precios se muestren predecibles expost.

Otra crítica a la hipótesis del mercado eficiente (HEM), es que supone agentes perfectamente racionales, es decir que buscan la maximización económica, más bien estos agentes actúan en función de elementos "psicológicos" (tales como las modas e imitaciones)

Por otro lado, la inversión de corto plazo es otro elemento que invalidaría la hipótesis del mercado eficiente, a través de la existencia de inversionistas especulativos de corto plazo. Estos inversionistas "apuestan" a que el mercado se equivoca de manera sistemática y tratan de obtener el máximo provecho de estos errores.

Según Leung, Daouk \& Chen (2000), la predicción de los retornos accionarios, dadas 


\section{Jorge Barrera H.}

las variables agregadas en el set de información de los inversores, es un hecho que es aceptado en la reciente literatura de las finanzas empíricas, y las preguntas apuntan hacia como usar la información de una manera óptima para predecir y transar en los mercados. No obstante el grado de predictibilidad de los retornos es generalmente considerado económicamente no significativo, por lo que Hodgson \& Nicholls (1991) sugiere evaluar la significancia económica de predecir la dirección de los cambios en los precios de los activos y no su nivel.

\section{FINANZAS CONDUCTISTAS}

Se puede definir a las finanzas conductistas como la integración de las economías clásicas o finanzas, con psicología y las ciencias de tomas de decisiones.

Las finanzas conductistas son un intento por explicar las causas de algunas anomalías que han sido observadas en los trabajos de investigación financiera y que están íntimamente ligadas con el estudio de cómo los inversionistas cometen errores de juicio sistemáticamente.

Los supuestos conductistas que se derivan del análisis de la hipótesis de mercados eficientes (HEM) puede ser expresado a través de 2 maneras, el que los inversionistas actúen imparcialmente para maximizar el valor de sus portafolios y el hecho que siempre actúen en su propio interés.

Otro aspecto de finanzas conductistas concierne al como los inversionistas forman sus expectativas sobre el futuro y como estas expectativas se transforman en precios seguros. Los retornos anormales asociados a ingresos no esperados, estrategias de corto y largo plazo son otros tópicos a tratar.

Existen dos factores que se observan en el comportamiento de la gente, que responden más que nada a sentimientos conductistas. Primero se encuentra el hecho que se presenta una conducta no maximizadora de riqueza, mediante esta idea se pretende rescatar el hecho que los inversionistas pretenden maximizar otras cosas que son más importantes para ellos que la riqueza, por lo que se puede inferir que no existe una vinculación tan estrecha entre el máximo de riqueza que se puede alcanzar y la máxima utilidad alcanzable, por parte del individuo que realiza la inversión.

De igual forma, se puede afirmar que los comportamientos en cierta forma parcializados, causan a los inversionistas o mejor dicho los llevan a cometer errores mentales sistemáticos como resultado de un incorrecto proceso de asimilación de la información que se 


\section{En el Perú se pueden modelar y predecir los rendimientos accionarios?}

tenía disponible para determinado momento del tiempo.

Se sabe que los conductistas sostienen que la hipótesis de eficiencia débil de mercado no se cumple, dado que existe un tipo de inversionista (noise-traders) el cual no realiza un análisis, que lo convencional diría racional, y en lugar de éste, realiza sus operaciones de compra y venta por entusiasmo o imitación, a su vez dada su "informalidad" realizan inversiones meramente especulativas en el corto plazo, aunque cabe destacar que dichos noise-traders, a pesar de la limitación de sus análisis obtienen beneficios con tan solo el uso de dichos indicadores.

Además, se debe tener especial cuidado con los precios dado que existen los fenómenos de sobre y sub-reacción; lo más saltante al respecto es que un hecho negativo produce una sobre reacción en sentido negativo y cuyo efecto es mayor al producido por un hecho bueno, el cual no tiene un efecto tan significativo.

Según la teoría de los mercados eficientes (HEM), también se puede generar sobre reacción y sub-reacción, la diferencia radica en que estas anomalías son igualmente de frecuentes, según dicho enfoque, a diferencia de los conductistas que sostienen que los fenómenos dependerán de las coyunturas que existan, así para un período la sub-reacción ante noticias negativas, dará la pauta del patrón de comportamiento, de igual forma ocurrirá en un mal período donde se sobredimensionará las noticias negativas, originando pérdidas, mayores a las que se deberían dar, según el contexto.

La dinámica social y la formación de precios, son aspectos que deben incorporarse como un elemento fundamental. Diversas actividades que se realizan están dadas por modas que se generan en el momento, el mercado de valores no se escapa a dichas "modas", por lo que diversas elecciones de los inversionistas, responden a éstas, por lo que las modas en un país se pueden estar moviendo en una dirección, mientras que en otro se mueven en dirección diferente, lo que tiene mucho que ver con la cultura del país.

\section{MODELOS NO LINEALES, DE ESTIMACIÓN DE DIRECCIÓN DEL CAMBIO}

La no linealidad inherente en los datos financieros y económicos ha sido observada por mucho tiempo; algunos investigadores han reconocido las limitaciones de técnicas econométricas que suponen una relación lineal como una aproximación. El supuesto de linealidad se ha utilizado en parte porque el coeficiente estimado de estos modelos es de fácil interpretación y porque la aplicación numérica era complicada en su momento. 


\section{Jorge Barrera H.}

Avances recientes en materia tecnológica computacional han relajado las restricciones o complejidades en calcular modelos y han llevado al desarrollo de técnicas econométricas no lineales como la regresión de regime-switching de Markov.

Además, investigadores en el mundo financiero recientemente han logrado otras aproximaciones y técnicas de estimación no lineales usadas en las ciencias físicas y biológicas, como son las redes neuronales artificiales (RNA) ${ }^{5}$ y los algoritmos genéticos.

Hodgson \& Nicholls (1991) sugieren evaluar la significancia económica de predecir la dirección de los cambios en los precios de los activos y no su nivel. En esta materia, Leung, Daouk \& Chen (2000) compararon la capacidad predictiva de los modelos de clasificación, no lineales ${ }^{6}$ con los de estimación de nivel, modelos lineales ${ }^{7}$ y concluyeron que los primeros se desempeñan mejor que los segundos en términos de su tasa de acierto (medida por el número de veces en que la dirección pronosticada es correcta) y, además, son capaces de generar beneficios económicos más altos.

a) Algoritmos Genéticos: Los algoritmos genéticos, introducidos por Holland (1975), consisten en una función matemática o una rutina que simula el proceso evolutivo de las especies, teniendo como objetivo encontrar soluciones a problemas específicos de maximización o minimización. Así, el algoritmo genético recibe como entrada una generación de posibles soluciones para el problema en cuestión, y arroja como salida los especimenes más aptos, para que se apareen y generen descendientes, los que deberían tener mejores características que las generaciones anteriores. Los algoritmos genéticos trabajan con códigos que representan las posibles soluciones al problema. Por ello, es necesario establecer una codificación. Al respecto, Davis (1994) se ala que la codificación más utilizada es la representación de las soluciones por medio de cadenas binarias.

Según Bauer (1994), este método puede ser utilizado fácilmente en aplicaciones financieras, tales como calificación de créditos bancarios, etc. y para reducir la complejidad y eliminar factores irrelevantes, para predecir índices de precios. ${ }^{8}$

Por otra parte, Feldman \& Treleaven (1994) se alaron que la mayor desventaja de los algoritmos genéticos es la dificultad que presentan para escoger una técnica de codificación manejable, y para determinar el tipo de selección y las probabilidades de los operadores genéticos, ya que no hay reglas fijas en esta materia.

b) Redes Neuronales Artificiales (RNA): Son modelos elaborados para simular el funcionamiento del cerebro $y$, en particular, la manera como éste procesa información. 


\section{En el Perú se pueden modelar y predecir los rendimientos accionarios?}

Dentro del contexto del análisis de series de tiempo se clasifican como modelos no lineales capaces de realizar conexiones entre los valores pasados y presentes de una serie de tiempo y extraer estructuras y relaciones escondidas que rigen el sistema de información. ${ }^{9}$

Muchos investigadores son atraídos actualmente por este enfoque, debido a que estos modelos (RNA) no están sujetos a supuestos restrictivos como la linealidad, y además tienen una gran capacidad para aprender, es decir, para identificar dependencias con base en una muestra finita, de manera que el conocimiento adquirido pueda ser generalizado a muestras no observadas (Herbrich et al, 1999). Asimismo, las RNA son instrumento importante en la modelación de variables en las cuales la existencia de un modelo estructural no es clara, pues no parten de supuestos a priori de los datos para el pronóstico y todo lo que de ellas puede decirse es inherente a las observaciones (Evans, 1997)

Herbrich et al (1999) se ala tres campos principales en los cuales se ha centrado la aplicación de estos modelos en la economía: Clasificación de agentes económicos, pronósticos de series de tiempo, modelaje de agentes con racionalidad limitada.

\section{CONCLUSIONES.}

La incapacidad de los modelos lineales tradicionales para ajustar y predecir el precio (random walk) y por ende la rentabilidad de la acción ha llevado a la búsqueda de nuevos modelos que sean capaces de percibir la dinámica de la serie. Ante esto surgen las redes neuronales artificiales (RNA), que tienen ventajas en varias dimensiones en relación con los métodos de análisis tradicionales.

Primero, poseen la capacidad de analizar y aprender rápidamente pautas complejas y con un alto grado de precisión.

Segundo, no están restringidas a la linealidad de la serie.

Tercero, las RNA tienen un buen rendimiento con datos incompletos, característica que se encuentra en la mayoría de los mercados no desarrollados, lo que amplía las ventajas de los estudios e investigaciones. 


\section{Jorge Barrera $\mathrm{H}$.}

\section{Notas Referenciales}

${ }^{1}$ B. . Malkiel, A Random Walk Down Wall Street, 2da edición universitaria, Nueva ork, Norton, 1981.

${ }^{2}$ Aznar A. \& Trívez F. "Métodos de Predicción en Economía” parte II., pp. 90-94.

${ }^{3}$ Por ejemplo, Lo \& Mackinlay (1988), registraron una correlación serial positiva; Conrad \& Kaul (1988, 1989), también encontraron evidencia de predictibilidad; DeBondt \& Thaler (1985), Fama \& French (1988), Poterba y Summers (1988) y Chopra, Lakonishok \& Ritter (1992), Jegadeesh (1990) hallaron correlación serial negativa y positiva para diferentes diferentes. Blume, Easley \& O Hara (1994) encontraron relaciones significativas entre diferentes rezagos.

${ }^{4}$ No siempre el uso racional de la información se traduce en un comportamiento aleatorio. Por ejemplo, un probable acuerdo de compra de una empresa elevaría el valor de sus acciones si existe una probabilidad (lejana) de que las negociaciones sean exitosas. Sin embargo, si no surgen desacuerdos, el precio de la acción subirá cuando el mercado re estime al alza las posibilidades de éxito del acuerdo. Ex-post el precio de la acción mostraría un comportamiento autocorrelacionado, a pesar de que el uso de la información fue eficiente.

5 "Artificiales" se usa a menudo para distinguir modelos matemáticos de redes neuronales con su contraparte biológica.

${ }^{6}$ Linear discriminant analysis, logit model y probabilistic neural network.

${ }^{7}$ Adaptive exponential smoothing, vector autoregression with Kalman filter, multivariate transfer function y multilayered feedforward neural network.

${ }^{8}$ Kim \& Ham (2000) mostraron que los algoritmos genéticos pueden ser usados para predecir índices de precios mejor que los métodos convencionales.

${ }^{9}$ Según Shahmurove y Witkowska (2000) 


\section{En el Perú se pueden modelar y predecir los rendimientos accionarios?}

\section{Bibliografía}

Aravena, J.C. (1998). Ajuste de los precios accionarios a la nueva información: caso mercado accionario chileno. Informe de memoria de título de Ingeniería Civil Industrial, Universidad de Concepción, marzo, pp. 38-72

Alexander, S. (1964) "Price Movements in Speculative Markets: Trenes or Random Walks, N 2" En: P. Cootner (ed.), The Random Character of Stock Market Prices. Cambridge, MA: MIT Press.

Allen, F. \& Karjalainen, R. (1999). Using genetic algoriths to find technical trading rules. Journal of Financial Economics, 51,245-271.

Arango, L. E. ; onzales, A. y Posada, C. E. (2002) "Returns and the Interest Rate: A Nonlinear Relationship in the Bogota Stock Market”. En: Banco de la República, Borradores de Economía, N 169

Bach, M., y E. Hansen (2002), "Estimación de redes neuronales con datos ultra frecuentes: Aplicación al mercado accionario chileno", Universidad de Chile

Bachelier, L. (1900). "Theory of Speculation". Cootner, P. (ed), En: The Random Character of Stock Market Prices, Cambridge, MA: MIT Press.

Box, .y Jenkins, . . (1976). Time Series Analysis: Forcasting and Control. Revised Edition. Holden Day.

Bauer, Richard J. Jr. (1994). enetic Algorithms and Investment Strategies, John Wiley \& Sons, Inc.

Berruecos, P. (2002). Evaluación de la Eficiencia Predictiva del Análisis Técnico en el Mercado Accionario Colombiano. Monografía de grado. Universidad EAFIT, Medellín.

Blume, L., Easley D. \& O Hara M. (1994). Market statistics and technical analysis: The role of volume. Journal of Finance 49,153-82

Campbell, J. ., A. W. Lo y A. C. Mackinlay (1997), the Econometrics of Financial Markets, Princeton, Princeton University Press.

Delgado , L. (1997) "El Mercado Bursátil Peruano y la Hipótesis del Mercado Eficiente”. Estudios Económicos BCRP.

Dominguez, N., Serrano, S. y Vi olas, P. (1997). "Mitos y Realidades de los Splits en la Bolsa Espa ola". Analisis Financiero. N 72, pp. 24-33.

El-Shazly, S., y H. El-Shazly (1999), "Forecasting Currency Forecasting Using Neural Net-works", ICL Systems Journal 11(2).

Fama, E. y French K. (1992) "The Cross Section of Expected Stock Returns". The Journal of Finance, N 2, junio, pp. 427-465. 


\section{Jorge Barrera H.}

Herbrich, R., Keilbach, M., raepel, T., Bollmann-Sdorra, P. \& Obermayer K. (2000). "Neural networks in economics: Background, applications and new developments". In T. Brenner, editor, Advances in Computational Economics: Computational Techniques for Modelling Learning in Economics, volume 11, pag 169-196. Kluwer Academics.

Johnson, C., y F. Soriano (2004), "Volatilidad del mercado accionario y la crisis asiática", El Trimestre Económico, vol.m L I, num. 282, pp. 355-388.

y R. Vergara (2004), "Monetary Policy in Chile: A Neural Network Approach", Escuela de Negocios Universidad Adolfo Ibá ezy Departamento de Economía, Universidad Católica de Chile, inédito.

Jung, J. y Shiller, R. J. (2002). One Simple tests of Samuelson s Dictum for the Stock Market. Cowles Foundation for Research in Economics, ale University. Discussion Paper N 1386.

Kanas, Angelos (2001). "Neural networks linear forecasts for stocks returns". International Journal of Finance and Economics, 6,245-254.

Kleiman, R. T; Payne, J. E. y Sahu, A. P. (2002) "Random Walks and Market Efficiency Evidence from International Real Estate Narkets". En: JRER. Vol. 24, N 3.

Parisi, a., Parisi, F. \& uerrero, J. L. (2003). "Modelos Predictivos de Redes Neuronales en Indices Bursátiles", El Trimestre Económico, México. (Forthcoming).

i, Min (2001). Predicting US recessions with leading indicators via neural netwok models. International Journal of Forecasting (17) 3,383-401.

Terrones Marco (1995) “Mercado de Capitales y Eficiencia” Notas para el Debate N 13.

Ross S., Westerfield R., Jaffe J. (1998). “Finanzas Corporativas” 3ra ed. pp.375-407

Shah, J., y M. Murtaza (2000), "A Neural Network Based Clustering Procedure for Bankruptcy Prediction”, American Business Review, 18(2), pp. 80-86.

White, H. (2000). "A reality check for data snooping". Econométrica. Vol. 68, N 5, 1097-1126.

Wu, C., y . Wang (2000), "A Neural Network Approach for Analyzing Small Business Lending Decisions", Review of uantitative Finance and Accounting 15 (3), pp. 259-276.

ilmaz, K. (2001). Market Development and Efficiency in Emerging Stock Markets. Koc University Istanbul, Turkey.

ablostky, E. (2001). Eficiencia del Mercado de Capitales. Una Ilustración. Universidad del CEMA, Buenos Aires. 